The Astrophysical Journal, 493:301-311, 1998 January 20

(C) 1998. The American Astronomical Society. All rights reserved. Printed in U.S.A.

\title{
IMAGING OF THE EGG NEBULA (CRL 2688) WITH WFPC2/HST: A HISTORY OF AGB/POST-AGB GIANT BRANCH MASS LOSS
}

\author{
Raghvendra Sahai, ${ }^{1}$ John T. Trauger,${ }^{1}$ Alan M. Watson, ${ }^{2}$ Karl R. Stapelfeldt, ${ }^{1}$ \\ J. J. Hester, ${ }^{3}$ C. J. Burrows, ${ }^{4}$ G. E. Ballister,${ }^{5}$ J. T. Clarke, D. Crisp, ${ }^{1}$ \\ R. W. Evans, ${ }^{1}$ J. S. Gallagher III, ${ }^{6}$ R. E. Griffiths, ${ }^{7}$ \\ J. G. Hoessel, ${ }^{6}$ J. A. Holtzman, ${ }^{2}$ J. R. Mould, ${ }^{8}$ \\ P. A. SCOWEN, ${ }^{3}$ AND J. A. WeSTPHAL ${ }^{9}$ \\ Received 1997 May 12; accepted 1997 August 14
}

\begin{abstract}
The proto-planetary nebula, CRL 2688, has been imaged through a wideband filter centered at 606 nm (F606W) with the Wide Field Planetary Camera 2 onboard the Hubble Space Telescope. CRL 2688 is the prototypical bipolar reflection nebula in which a star is surrounded by a dense, flattened cocoon of dust seen nearly edge-on and starlight escapes preferentially along the polar directions producing a pair of bright nebulosities, one above and one below the equatorial plane. We find a pair of radial "searchlight beams" emerging from within the dusty cocoon which intersect at the position expected for the central star when extrapolated inside the cocoon. The beams are crisscrossed by a large number of roughly round arcs with their center of curvature in the vicinity of the central star. The arcs are not systematically elongated along the polar axis of the nebula, as would be expected in the current model of CRL 2688 where the nebular density decreases with latitude.

Our image directly shows the last $\sim 13,000$ yr history of mass ejection from the central star while it was on the tip of the asymptotic giant branch (AGB). We find that the average surface brightness varies as $r^{-3.7}$, implying that the average mass-loss rate or the scattering opacity of the dust grains varies as $r^{-0.7}$, i.e., one or both of these parameters have steadily increased with time. The temporal resolution of $\approx 25 \mathrm{yr}$ in our images has provided direct evidence for episodic increases in the mass-loss rate by factors of $\sim 2$ or more, occurring every $150-450 \mathrm{yr}$, and lasting over periods of 75-200 yr. These irregularities in the mass-loss rate are roughly spherically symmetric. We have resolved the edges of the searchlight beams, as well as the peculiar structure of the inner region of the nebula $\left(1 . .5<\right.$ radius $\left.<6^{\prime \prime}\right)$. Our data require a new model for CRL 2688 in which the beams result from starlight escaping through a pair of nonuniform annular holes in the dust cocoon which are coaxial with the polar axis of the nebula. The holes have probably been generated by a young (less than $200 \mathrm{yr}$ ) high-velocity outflow which streams out through these holes and interacts with the surrounding dense AGB wind to produce the peculiar structure of the inner nebula. The cocoon contains dust grains of size about $0.6 \mu \mathrm{m}$, significantly larger than those in the extended nebula.
\end{abstract}

Subject headings: ISM: individual (CRL 2688) - ISM: jets and outflows - ISM: structure reflection nebulae - stars: AGB and post-AGB — stars: mass loss

\section{INTRODUCTION}

It is widely accepted that planetary nebulae evolve from asymptotic giant branch (AGB) stars, but the details of the transition, which occurs very rapidly $(\sim 2000 \mathrm{yr})$, are very poorly understood. During this phase the central star's tem-

\footnotetext{
${ }^{1}$ Jet Propulsion Laboratory, California Institute of Technology, 4800 Oak Grove Drive, Pasadena, CA 91109.

${ }^{2}$ Lowell Observatory, Mars Hill Road, Flagstaff, AZ 86001.

${ }^{3}$ Department of Physics and Astronomy, Arizona State University, Tyler Mall, Tempe, AZ 85287.

${ }^{4}$ Space Telescope Science Institute, 3700 San Martin Drive, Baltimore, MD 21218.

${ }^{5}$ Department of Atmospheric, Oceanic, and Space Sciences, University of Michigan, 2455 Hayward, Ann Arbor, MI 48109.

${ }^{6}$ Department of Astronomy, University of Wisconsin, Madison, 475 North Charter Street, Madison, WI 53706.

${ }^{7}$ Department of Physics, Carnegie Mellon University, Pittsburgh, PA 15213-3890.

${ }^{8}$ Mount Stromlo and Sidings Springs Observatories, Australian National University, Weston Creek Post Office, ACT 2611, Australia.

${ }^{9}$ Division of Geological and Planetary Sciences, California Institute of Technology, Pasadena, CA 91125.
}

perature rises from about $2500 \mathrm{~K}$ to $30,000 \mathrm{~K}$, leading to the genesis of a luminous planetary nebula. Objects in this transitional phase, known as proto-planetary nebulae, are the key missing link in our understanding of late stellar evolution. The most well-known proto-planetary nebula is the Egg Nebula in Cygnus (CRL 2688), first discovered on the Palomar Observatory Sky Survey as a small-egg-shaped nebulosity (Ney et al. 1975). It is also one of the most prominent members of the well-known class of bipolar reflection nebulae, in which a star (either pre-main-sequence or postmain-sequence) is surrounded by a dense, flattened cocoon of dust seen nearly edge on. Starlight escapes preferentially along the polar directions, illuminating material above and below the equatorial plane which appears as a pair of bright nebulosities. The post-AGB illuminating star in CRL 2688 has an F5 Ia spectrum. CRL 2688 has been studied at optical (Ney et al. 1975), infrared (Latter et al. 1993, hereafter LHK93), and millimeter wavelengths (Truong-Bach et al. 1990; Young et al. 1992; Jaminet et al. 1992; Yamamura et al. 1995) from the ground. The distance to this object is not well known, and we assume the commonly used value of $1 \mathrm{kpc}$. In this paper, we present the first high-resolution 
images of CRL 2688 taken from space using the Hubble Space Telescope $(H S T) .{ }^{10}$

\section{OBSERVATIONS AND RESULTS}

Using the Wide Field Planetary Camera 2 (WFPC2) onboard HST (Trauger et al. 1994), we have imaged CRL 2688 through a wideband filter centred at $606 \mathrm{~nm}(\mathrm{~F} 606 \mathrm{~W}$, $\langle\lambda\rangle=593.5 \mathrm{~nm}, \delta \lambda=149.7 \mathrm{~nm}$; Biretta et al. 1996). The data, taken on 1995 July 17 are comprised of a single $260 \mathrm{~s}$ exposure. Standard flat fielding and bias subtraction were performed on the image. Cosmic rays were removed by scanning through the image with a $5 \times 5$ pixel window, determining all pixels which were $3 \sigma$ above the median value, and replacing them with nearest-neighbor interpolated values. All detectable nebulosity lies in the field of the WFPC2 camera 3, which has a plate scale of 0.0996 pixel $^{-1}\left(100 \mathrm{AU}\right.$ or $1.5 \times 10^{15} \mathrm{~cm}$ at a source distance of 1 $\mathrm{kpc})$.

The $\mathrm{F} 606 \mathrm{~W}$ image is shown in reverse gray scale in Figure 1 $a$ (Plate 8); however, in the following description, the terms "bright" and "dark" will carry their usual meaning). The image covers the central $745 \times 745$ pixels of the original $800 \times 800$ image. Small sections (738 pixels) of the very bright inner regions are saturated. Most of the saturated pixels are located in the northern lobe. The notable morphological features of the nebula are two pairs of radial "searchlight-beam" features emerging from a dark lane, which are presumably due to an edge-on flattened obscuring structure in the center (hereafter referred to as the dust cocoon). The major symmetry axis of the nebula bisects the dark, "shadow" region between the searchlight beams. The beams are crisscrossed by a large number of round arcs. The arcs are also seen faintly in other regions of the nebula, at lower latitudes (with the polar axis being defined as the axis bisecting the angle between the searchlight beams). The brightest parts of the searchlight-beam features, and small sections of a few (three) of the most prominent circular arcs within the region of the searchlight beams, had been seen faintly in ground-based images by LHK93 and Crabtree \& Rogers (1993). Our WFPC2 images show that the arcs are very numerous and are present throughout the visible nebula. We have resolved the structure of the arcs and the searchlight beams.

An image of the small-scale structure in which the low spatial frequency structure has been suppressed has been made by subtracting out an "unsharp mask" image from the raw image (Fig. 1b, Pl. 9). The unsharp masked image was generated by smoothing the logarithmic intensity image in Figure $1 a$ as follows. The intensity for any pixel was replaced by the 30th percentile value of all intensity values in a circular sample area with a 10 pixel radius centred on the reference pixel. This image shows that the searchlight beams have very straight and sharp edges. The beams in the southern lobe are more sharply defined. We find that the position angles of the intensity-weighted centers of the beams in the north lobe (south lobe) are 31.4 (212.1) and $46^{\circ} .1$ (230.9) counterclockwise from the horizontal axis in the image (for reference, north is inclined at an angle of 21.83 clockwise from the horizontal axis). Hereafter, we will use these inclination angles to refer to the

\footnotetext{
10 The NASA/ESA Hubble Space Telescope is operated by AURA, Inc., under contract NAS 5-26555.
}

beams individually. Thus the beams in the north lobe are close to, but not precisely, colinear with their counterparts in the south lobe. Extrapolating the beams from each lobe inside the dark lane, we find that they intersect at a position intuitively expected for the central star; we have used this location as the central star position $\left(\alpha_{\mathrm{J} 2000}=21^{\mathrm{h}} 02^{\mathrm{m}} 18.27\right.$, $\left.\delta_{\mathrm{J} 2000}=36^{\circ} 41^{\prime} 37^{\prime \prime} .0\right)^{11}$. Faint radial striations can be seen within the searchlight beam region. These striations have low contrast. One of the most prominent of these can be seen extending for a long way (from a radius of $11^{\prime \prime}$ to $25^{\prime \prime}$ ) within the $46^{\circ}$ searchlight beam in the northern lobe.

The detailed morphology of the very bright inner regions where the searchlight beams emerge from the dark lane can also be seen clearly for the first time in the WFPC2 image (Fig. 1c [Pl. 10]). At the base of the searchlight beams, there are bright spindle-shaped regions. In the southern lobe, the southern edges of the spindle are tapered inward, away from the linearly diverging outer edges of the beams. The spindle is bifurcated by the inter-searchlight dark "shadow" region which extends inward to the dark lane. A significant portion of the northern spindle is saturated, so its actual shape cannot be determined. The morphology of the interfaces between the dark lane and the southern spindle is distinctly different from that in the north. In the south, the spindle juts against a smooth extended edge which bulges outward slightly with respect to the center of the nebula. In contrast, the northern spindle steadily becomes narrower in width approaching the dark lane, culminating in an apex which has the shape of the letter $W$ with its symmetry axis aligned with the polar axis.

The most distant nebulosity in our image, detected at a level of $24.4 \mathrm{mag} \mathrm{arcsec}-2$, lies on the northern side of the nebula at $51^{\prime \prime}\left(7.6 \times 10^{17}\right.$ at $\left.1 \mathrm{kpc}\right)$ from the center which is significantly larger than seen in previous work. For example, the deep I-band image published by LHK93 shows nebulosity extending up to a maximum of about $27^{\prime \prime}$ from the center. Truong-Bach et al. (1990) mapped circumstellar CO millimeter-wave emission in this object with $12^{\prime \prime}$ resolution, and their deconvolved outer radius is a factor 3.3 smaller than our value. Determining the maximum linear extent of the nebula is crucial since the total nebular mass, for a constant mass-loss rate, is directly proportional to its size. Although most models of the mass-outflow in CRL 2688 have invoked the usual assumption that the mass-loss rate has remained constant with time, we show below that it is likely that the mass-loss rate in CRL 2688 has, on the average, increased with time.

\section{THE ARCS}

The arcs appear roughly round but tend to disappear near the equatorial plane, presumably as a result of lack of illumination by direct starlight. Several of the arcs appear to intersect. The arcs are probably not two-dimensional structures but rather the limb-brightened surfaces of thin threedimensional roughly spherical shell-like structures around the central star. These shells appear to be incomplete since, in general, none of the arc structures can be traced for a full $360^{\circ}$ all the way around the center (allowing for the gaps at low latitudes around the equatorial plane, where there is very little illumination); we have verified this by overlaying

\footnotetext{
${ }^{11}$ Average pointing errors in coordinates derived from HST images are about 0 ".9.
} 
circles of varying radii around the center. The thicknesses of the shells must be smaller (by a factor $\approx 1.5-2$ ) than the thicknesses of the arcs, which range from about 0 ".5 to 1 .'2, i.e., $(0.75-1.8) \times 10^{16} \mathrm{~cm}$ at $1 \mathrm{kpc}$. Assuming the outflow velocity of dust in the arcs and the gas in the large-scale molecular envelope to be the same $\left(20 \mathrm{~km} \mathrm{~s}^{-1}\right)$, we find that the arcs represent enhancements in the average local density of the mass outflow occurring over periods of about 75-200 yr. These enhancements have apparently occurred throughout the $12,700 \mathrm{yr}$ old $\left(=7.6 \times 10^{17} \mathrm{~cm} / 20 \mathrm{~km} \mathrm{~s}^{-1}\right)$ mass-loss history of CRL 2688 seen in the WFPC2 image. The radial separation between the most prominent arcs ranges from about 0 ".6 to 1.8 , representing timescales of $150-450 \mathrm{yr}$. Radial cuts of the intensity (for a set of closely spaced latitudinal angles) spanning a $14^{\circ}$ wide region of the $31^{\circ}\left(212^{\circ}\right)$ searchlight beam of the northern (southern) lobe, are shown in Figure 2a. Positive (negative) values of radius refer to the northern (southern) lobe. Each intensity trace has been normalized by the peak value of the intensity, and then shifted vertically for clarity. Beyond the central region $(r>6$ " and $r<-3^{\prime \prime}$ ), the arcs appear as small local peaks superimposed on a smoothly decreasing background. Some of the peaks appear at the same radius in all of the traces, and thus represent an arc which is locally circular, while other peaks shift their radial position steadily, representing departures from circularity. Other peaks appear in only two or three neighboring traces. No clear correspondence can be found in the radial distribution of peaks on either side of the center (Fig. 2b). Radial distributions of the scattered light on diametrically opposed sides of the nebula, averaged over a $60^{\circ}$ latitude range which avoids (1) the dark-lane region near the equator and (2) regions with very saturated stellar sources which are difficult to remove from the image, show a higher degree of correspondence of the peaks (Fig. 2c). More arcs can be seen in the northern lobe, compared to the southern one. This is probably a sensitivity affect, since the northern lobe is brighter than the southern one by about a factor 4-5.

The most direct argument for why the arcs cannot be light echoes propagating out through the nebula resulting from variations in the stellar light, is that the locations and shapes of the brightest arcs in the HST image are the same as seen in ground-based images from earlier epochs (e.g., I-band image in LHK93, visual images by Crabtree \& Rogers 1991, 1993). ${ }^{12}$ The arcs must be long-lived rather than transient structures. This is because the density fluctuations responsible for the arcs, once created, get "frozen" into the envelope structure since the sound speed is small compared to the outflow speed of $20 \mathrm{~km} \mathrm{~s}^{-1}$ throughout the nebula. Specifically, the gas temperature falls rapidly below $100 \mathrm{~K}$ for angular radii larger than 0.3 (Truong-Bach et al. 1990), giving a sound speed, $c_{s}=0.76 \mathrm{~km} \mathrm{~s}^{-1}(T / 100 \mathrm{~K})^{1 / 2}$ $\left[c_{S}=(\gamma k T / \mu)^{1 / 2}\right.$, where $\lambda=5 / 3$, is the ratio of the specific heats at constant pressure and constant volume at the relatively low temperatures in the nebula (less than $100 \mathrm{~K}$ ), and $\mu=m\left(\mathrm{H}_{2}\right)$ is the mean molecular weight].

The brightness of each arc, relative to the brightness of the nebula in neighboring regions (typically a factor $1.5-2$ ),

\footnotetext{
${ }^{12}$ We have obtained the original $V$-band image presented by Crabtree $\&$ Rogers and compared it with our HST image, degraded to a typical ground-based 1 " resolution. We find that we can match several bright arc-like features in both images. The brightness variations in the images are sufficiently distinctive that there is no ambiguity in identifying corresponding features in both images.
}

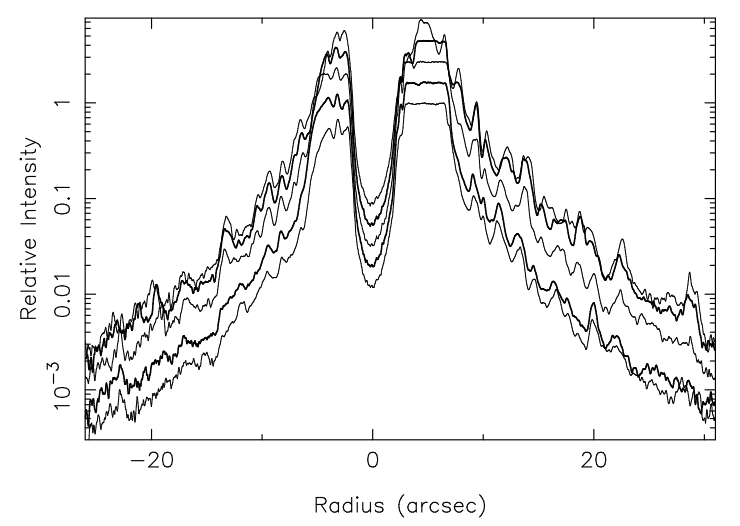

FIG. $2 a$

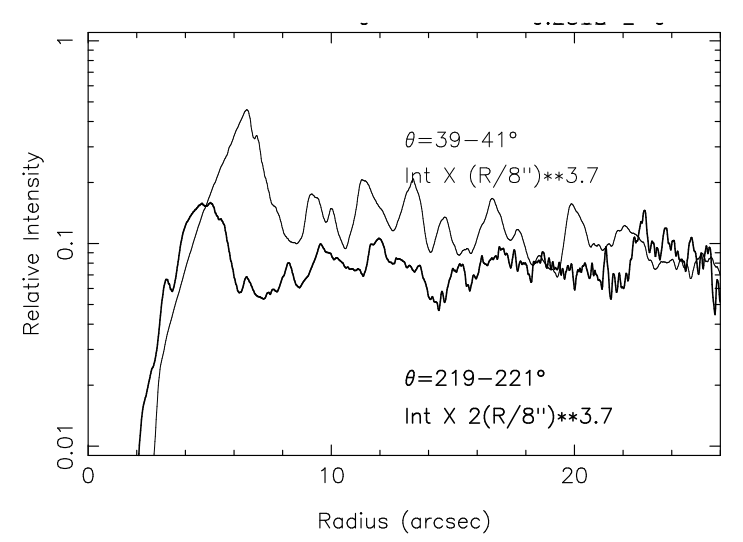

FIG. $2 b$



FIG. $2 c$

FIG. 2.- (a) Radial cuts of the intensity taken through the center of the CRL 2688 nebula shown in Fig. $1 a$, for a set of closely spaced latitudinal angles which traverse a $14^{\circ}$ wide region in the $31^{\circ}$ and $212^{\circ}$ searchlight beams. Positive (negative) values of radius refer to the $31^{\circ}\left(212^{\circ}\right)$ beam. Each intensity trace has been normalized by the peak value of the intensity and then shifted vertically for clarity. Beyond the central region $\left(r>6^{\prime \prime}\right.$ and $\left.r<-3^{\prime \prime}\right)$, the arcs appear as small local peaks superimposed on a smoothly decreasing background. Some of the peaks appear at the same radius in all of the traces, and thus represent arcs which are locally circular, while other peaks shift their radial position steadily, representing departures from circularity. Other peaks appear in only two or three neighboring traces. (b) Overlays of intensity traces taken along the same radial vector passing through the center, but on opposite sides of the nebula, showing the lack of any obvious radial alignment of the peaks. The intensity has been scaled by $r^{3.7}$ to flatten the curves for better comparison. (c) Latitudinally averaged radial distribution of the scattered light for the northern and southern lobes. The latitudinal averages avoid the angular region near the equator (and regions with very saturated stellar sources which are difficult to remove from the image). The ordinate is intensity, scaled by $r^{3.7}$, and the flat curves demonstrate that the intensity decreases with radius as an $r^{-\alpha}$ power law for $r>9^{\prime \prime}$, with $\alpha \approx 3.7$. 

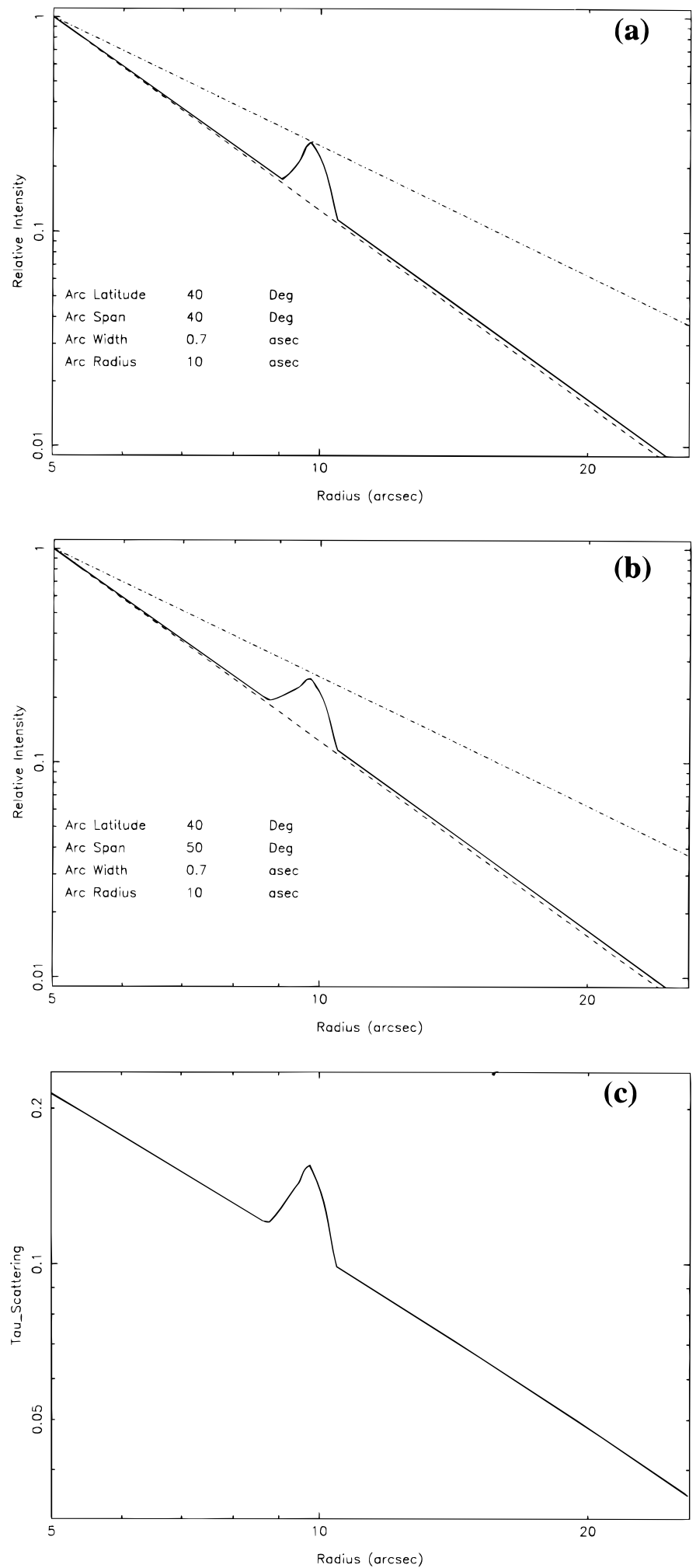

FIG. 3.-Radial decrease in intensity from a spherically symmetric model nebula with an inverse-square radial decrease in density with $(a)$ and without $(b)$ a latitudinally increasing illumination from a central star. The dashed and the dot-dashed curves, respectively, show $r^{-3}$ and $r^{-2}$ variations. The corresponding line-of-sight tangential optical depth is shown in (c). A three-dimensional incomplete shell (lying on the surface of a sphere around the central star), characterized by a higher-than-average (by a factor 3) local density has been included in the model to simulate an arc which is a factor 2 brighter than the surrounding nebulosity. The models have been computed for an optically thin nebula, with an optical depth of 0.1 in the vicinity of the arc. The actual optical depth in CRL 2688 is probably several times larger, requiring more than a factor 3 density enhancement in the shell producing the arc. can be used to derive the localized increase in optical depth within the arcs, provided we know whether the nebula is optically thin $\left(\tau_{S} \ll 1\right)$ or thick $\left(\tau_{S} \gg 1\right)$ to scattered light. In an optically thin, spherically symmetric nebula, with a radial density variation, $\rho(r)$ given by $r^{-\alpha}$, the scattered light, $S(r)$, varies as $r^{-(\alpha+1)}$ (e.g., Tamura et al. 1988), assuming no change in the scattering properties of the grains. In CRL 2688, the assumption of spherical symmetry is violated since the scattered light varies as a function of latitudinal angle, either as a result of a latitudinal variation of (1) the nebular density or of (2) the incident starlight. By carrying out a simple numerical integration (e.g., Morris 1981), we have calculated the radial distribution of scattered light in the presence of latitudinal variations of the nebular density or the incident starlight (Fig. 3). In both cases, we find that if $\rho(r) \propto r^{-2}$, then $S(r) \propto r^{-(\alpha+1)}$ with $\alpha \leq 2$; an increase in the optical depth makes $\alpha$ smaller. If the nebula is optically thick $\left(\tau_{s} \gg 1\right)$, the radial variation of the surface brightness depends not only on singly but also on multiply scattered photons and, therefore, cannot be calculated without detailed modeling. Using a Monte Carlo multiplescattering code (Watson 1994), we find that in a model with $\rho(r) \propto r^{-2}$, and (say) $\tau_{S} \sim 2$ at a radius $r=10^{\prime \prime}, \alpha \approx 1$. Our observations show that for both the northern and southern lobes in CRL 2688, the radial distribution of the scattered light follows an $r^{-(\alpha+1)}$ power law for $r>9^{\prime \prime}$, with $\alpha \approx 2.7$ (Fig. 2b, 2c). We conclude, therefore, that either (1) the underlying nebular density or (2) the scattering opacity of the dust grains varies at least as fast as $r^{-2.7}$. If possibility (1) is correct, then, since $\rho(r)=d M / d t /\left[4 \pi r^{2} V_{e}(r)\right]$, either the mass-loss rate $d M / d t$ has decreased with radius, or the outflow velocity $V_{e}(r)$ has increased with radius. We rule out the latter possibility because it implies a significant increase in the full width at zero intensity (FWZI) of nebular molecular lines as a function of radial distance, contrary to observations. For example, we can compare the $\mathrm{CO}$ millimeter-wave line profiles at $12^{\prime \prime}$ in Truong-Bach et al. (1990) (or 15"-17" in Kawabe et al. 1987) with those at twice these offsets (profiles labeled 24" by Truong-Bach et al. or $30^{\prime \prime}-42^{\prime \prime}$ by Kawabe et al.). If $V_{e}(r)$ varied as $r^{0.7}$, the FWZI of the profiles at the larger distance would be about 1.5 times larger, which is clearly not supported by the data. An increasing mass-loss rate with time has been independently proposed by Yamamura et al. (1996) based on ${ }^{13} \mathrm{CO}$ $(J=1-0)$ millimeter-wave line emission maps of the gas distribution (with 5".5 resolution) in CRL 2688.

Thus, is appears most likely CRL 2688 has undergone a steadily increasing average mass-loss rate. In order to estimate the total envelope mass, we take the mass-loss rate $\left(1.7 \times 10^{-4} M_{\odot} \mathrm{yr}^{-1}\right)$ derived from the $\mathrm{CO}$ data assuming it to be constant with time (Truong-Bach et al. 1990) to represent the correct mass-loss rate at a radius equal to half of the total extent of the envelope seen in $\mathrm{CO}$ emission. The total mass in the visible envelope, $M_{\text {tot }}$, for $\rho(r) \propto r^{-2.7}$, given by $\left(d M_{0} / d t\right)\left(r_{\max }^{0.3} r_{0}^{0.7} / V_{\text {exp }}\right)$, where $d M_{0} / d t$ is the massloss rate at radius $r_{0}=10^{\prime \prime}$, is $0.7 M_{\odot}$, thus implying a main-sequence stellar mass of about $1.3 M_{\odot}$ (since most white dwarf masses lie in a relatively narrow range of about 0.5-0.6 $M_{\odot}$ ). Alternatively, if the mass-loss rate was constant, then the total mass of the envelope would be $2.1 M_{\odot}$, giving a main-sequence stellar mass of about $2.7 M_{\odot}$. Note that since we are sensitivity limited in detecting the faint outer regions of the nebula in the HST image, the above estimates of the total envelope mass are strictly lower limits; 
however, for the case of increasing mass-loss rate, the sensitivity to the outer radius is much less than in the case of a constant mass-loss rate.

In the optically thin approximation $\left(r_{S} \ll 1\right)$, our modeling shows that a shell, characterized by a density higher by a factor 3 than the average density at that radius, produces an arc which is slightly more than a factor 2 times brighter than the underlying nebular brightness at that point (Fig. 3) (if $\tau_{S} \gg 1$, a significantly smaller contrast in arc/nebular brightness is obtained; e.g., in a region where $\tau_{S}=1$, a thin shell of material with a factor 3 density enhancement produces an intensity enhancement of only a factor 1.3). The differential increase in brightness is less than the differential increase in the local density because of the effect of projection of the three-dimensional shell surface. The increase in brightness depends also on the angular span of the arc for small spans and, in the case of a latitudinally varying density or incident starlight, on the latitude of the arc location.

Is the CRL 2688 nebula optically thin $\left(\tau_{S} \ll 1\right)$ or thick $\left(\tau_{S} \gg 1\right)$ to scattering? We use the long-wavelength optical depth derived from thermal dust emission to answer this question since it is independent of the distance, $D$, and the absolute value of the emissivity $\kappa$, both of which are poorly known (e.g., eq. [A5] of Sahai 1990; Jura 1986). ${ }^{13}$ Working backward from the average dust mass-loss rate in CRL 2688 derived from flux measurements at $400 \mu \mathrm{m}-2 \mathrm{~mm}$ (1.4$2.3 \times 10^{-6} M_{\odot} \mathrm{yr}^{-1}$; Sopka et al. 1985; Knapp, Sandell, $\&$ Robson 1993), and the $400 \mu \mathrm{m}$ dust opacity used for the derivation $\left(20-35 \mathrm{~cm}^{2} \mathrm{~g}^{-1}\right)$, we find that at $\varphi=10^{\prime \prime}$, $\tau(400 \mu \mathrm{m})=\left(\kappa d M_{d} / d t\right) /\left(4 \varphi D V_{\text {exp }}\right) \sim 0.0026$, giving $\tau_{s}(0.6$ $\mu \mathrm{m}) \sim 0.5$, assuming (1) the dust absorption opacity, $\kappa_{a}(\lambda) \propto \lambda^{-p}$, with $p=0.8$ (e.g., Knapp et al. 1993) and a typical albedo of 0.5 at $0.6 \mu \mathrm{m}$, and (2) the distance, $D=1$ kpc. The above analysis assumes that most of the longwavelength emission comes from dust distributed in a spherical, inverse-square density envelope and is not, for example, concentrated in an equatorial disk, which would make it impossible to infer optical depths in the nebular lobes from the long-wavelength fluxes of the whole object. A map of the dust emission at $2.6 \mathrm{~mm}$ shows a roughly round source with a sensitivity limited size of about 10" (Yamamura et al. 1995). A Very Large Array (VLA) 8.4 $\mathrm{GHz}$ map shows extended emission out to a radius of at least $15^{\prime \prime}$; the map is inconsistent with a disklike morphology (Knapp et al. 1994). In summary, it appears that most of the extended nebular region in CRL 2688 has a modest scattering optical depth at $0.6 \mu \mathrm{m}$.

\section{OUTER AND INNER REGIONS OF CRL 2688}

\subsection{The Outer Region ( $\left.r>6^{\prime \prime}\right)$ of the Nebula: The Searchlight Beams}

What is the nature of the searchlight beams? We first examine the existing model based on analysis of groundbased images. In early photographic images, the "searchlight-beam" features appeared as small protrusions on the main reflection lobes and could be modeled as the region where the emergent light reaches its maximum intensity after being scattered in a density distribution which

\footnotetext{
${ }^{13}$ We are assuming that the dust-gas drift velocity is small compared to the total outflow velocity, which is certainly the case for at least most of the extended envelope in CRL 2688.
}

peaks in the equatorial plane, decreasing monotonically with latitude, dropping sharply to zero near the polar region (i.e., latitude $\theta \approx 80^{\circ}$; Morris 1981 ; Yusef-Zadeh, Morris, \& White 1984; LHK93). This model (hereafter YMW model) was successful in providing a simple framework for interpreting a variety of bipolar reflection nebulae.

However, our WFPC2 image shows that the density distribution in the YMW model cannot be correct in the case of CRL 2688 for several reasons. First, our image does not show any evidence of systematic ellipticity in the arcs as would be expected in the YMW model because of the density-dependent drift velocity of the dust grains through gas. Under stellar radiation pressure, dust grains would drift through the gas at a velocity $V_{d}$ which would increase monotonically with latitude because $V_{d}=$ $\left(L V_{e}\langle Q\rangle / c d M / d t\right)^{1 / 2}$, and in the YMW model $d M / d t$ decreases with latitude, whereas $\langle Q\rangle$ increases with latitude ( $L$ is the stellar luminosity, $\langle Q\rangle$ is the ratio of the frequencyintegrated radiation absorption cross section to the geometric cross section, $d M / d t$ is the gas mass-loss rate, and $V_{e}$ is the gas outflow velocity). Using the variation of $V_{d}$ with latitude in CRL 2688 as computed by Jura \& Kroto (1990) (who have used an approximation to the latitudinal variation of density from the YMW model), we find that the dust in shells which were spherical at ejection would deform into elliptical shapes wih the major axis aligned with the polar axis and a major-to-minor axis ratio of about 1.65-1.3 for grains of radius $0.1-0.01 \mu \mathrm{m}$. Such a pattern is clearly not observed for the arcs.

Second, the YMW model does not account for the very sharp edges of the "searchlight beams" (especially when multiple scattering is taken into account). Nor does it account for faint radial striations seen within the searchlight beam region in our HST image. These striations have low contrast and are best seen in Figure $1 b$, the most prominent one lying inside the $46^{\circ}$ searchlight beam. In order to quantitatively demonstrate the discrepancy between the YMW model and the HST data, we have computed, using our own multiple-scattering code (Watson 1994), the nebular light distribution assuming the same parameters for the density distribution and dust scattering properties as LHK93. Our multiple-scattering code has been tested extensively, and the results from test cases agree with analytical models. We find that there are systematic differences between the model intensity (using the LHK93 physical parameters) and our data. In Figure 4 we show a comparison of the observed intensity as a function of angular offset generated from a polar cut across the searchlight beams in the northern lobe. First, the change in brightness as a function of angular offset across the searchlight-beam features in the data is much more steep than in the model. Second, the observed ratio of the peak intensity to that in the shadow region between the beams is much larger than in the model. The same discrepancies are found to exist for the searchlight beams in the southern lobe. Although we have assumed an isotropic phase function, it is unlikely that departures from this assumption can alleviate these discrepancies since the scattering angles from the material in the intersearchlight and searchlight-beam regions are very similar. It is clear that the earlier models succeeded in providing reasonable fits to the ground-based images with seeing-limited resolution of $1^{\prime \prime}-2^{\prime \prime}$ because the brightness contrast between searchlight beams and the surrounding nebulosity was significantly reduced as a result of the seeing. 




Fig. 4.-Intensity as a function of angular offset, generated from a polar cut taken through the searchlight beams in the northern lobe of CRL 2688 in our F606W WFPC2 image (dashed curve). The polar axis corresponds to an angular offset of $39^{\circ}$. Also shown, overlaid on the data, is the corresponding model intensity (solid curve) calculated using a multiplescattering Monte Carlo code, based on the YMW physical structure and parameters for CRL 2688 given by LHK93. There are large systematic differences between the two, indicating that the YMW model of CRL 2688 is not correct.

No evidence of the expected biconical polar holes in the YMW model can be seen in interferometric maps of molecular line emission. For example, Bieging \& Nguyen-Q-Rieu (1996) find no evidence for absence of gas in the polar directions in their $3.5 \times 3$. 0 resolution map of millimeter-wave $\mathrm{HCN}$ line emission. Bright $\mathrm{HCN}$ emission at the radial velocity of the star (which provides a view of gas expanding in the plane of the sky) is clearly seen in the northern and southern lobes along the polar axis. Bieging \& Nguyen- $Q$ Rieu (1996) conclude that their data pose a challenge to the latitude dependence of the envelope matter distribution as inferred from the YMW-type dust scattering models.

Finally, in the YMW model, it is necessary to set the scattering optical depth to be roughly the same at all wavelengths in the $0.8-2.2 \mu \mathrm{m}$ range in order to reproduce the relatively wavelength-insensitive overall bipolar morphology of the nebula observed (LHK93). However, in contradiction, near-IR polarization studies of the extended CRL 2688 nebula show that the dominant grains have a radius $\sim 0.07 \mu \mathrm{m}$, implying a strong dependence of opacity on wavelength.

\subsection{The Inner Region $\left(r<6^{\prime \prime}\right)$ of the Nebula}

The detailed morphology of the inner regions of CRL 2688 is also at odds with the YMW model. First, in this model, it is not possible to even qualitatively account for the spindle-shaped bright region in the southern lobe, with its outer edges curving inward, away from the linearly diverging outer edges of the searchlight beams. Second, the shapes of the interfaces between the dark lane and the nebular lobes seen in our HST image are qualitatively different from those found in the YMW model. In the YMW model (see, e.g., Fig. $9 a$ of LHK93), the interfaces are similar, with prominent, extended, nearly flat portions orthogonal to the polar axis: the extent of the flat part is larger for the brighter lobe. In contrast, our observations show that the fainter (southern) lobe has an extended nearly flat interface with a much larger extent (orthogonal to the polar axis) than the interface in the north (which is not even flat, but is shaped like the letter W).

In summary, we conclude from the arguments given above that a YMW-type model is not valid for CRL 2688 and propose a new model.

\section{A NEW MODEL}

We propose that the detailed morphology of the CRL 2688 results primarily from anisotropic illumination of nebular dust by starlight, escaping from annular holes in a flattened dust cocoon surrounding the star (shown schematically in Fig. 5). The holes must be annular rather than circular in order to produce the dark intersearchlight shadow region. The cocoon is tilted slightly with its southern edge nearer to us. In marked contrast to the YMW model, where there is a physical continuity between the high-latitude and low-latitude dust, the dust cocoon in our model is a compact physical structure, different from the surrounding nebula. This idea has important implications for the formation of planetary nebulae which we discuss in $\S 6$. Our picture of the dust cocoon is supported by the ${ }^{13} \mathrm{CO}(J=1-0)$ study of the gas distribution in CRL 2688 by Yamamura et al. (1996), who conclude that "no large-scale axisymmetric structure larger than $8 \times 10^{16}$ $\mathrm{cm}$... is present in the envelope of CRL 2688."

All of the difficulties encountered by the YMW model are resolved by our model for the searchlight beams. The sharp edges of the beams simply result from correspondingly sharp edges in the cavities in the dust cocoon through which the starlight escapes. The morphology of the nebula does not change significantly with wavelength in the $0.8-2.2$ $\mu \mathrm{m}$ range (LHK93) since the latitudinal distribution of light is largely a geometric effect of light escaping through the annular holes and being scattered into the line-of-sight. The dark region between the beams is due to an absence of light, not of matter, hence the millimeter-wave emission maps do not show a local minimum in this region. The faint radial striations result from shadows cast by blobs of material distributed within the region of the annular holes.

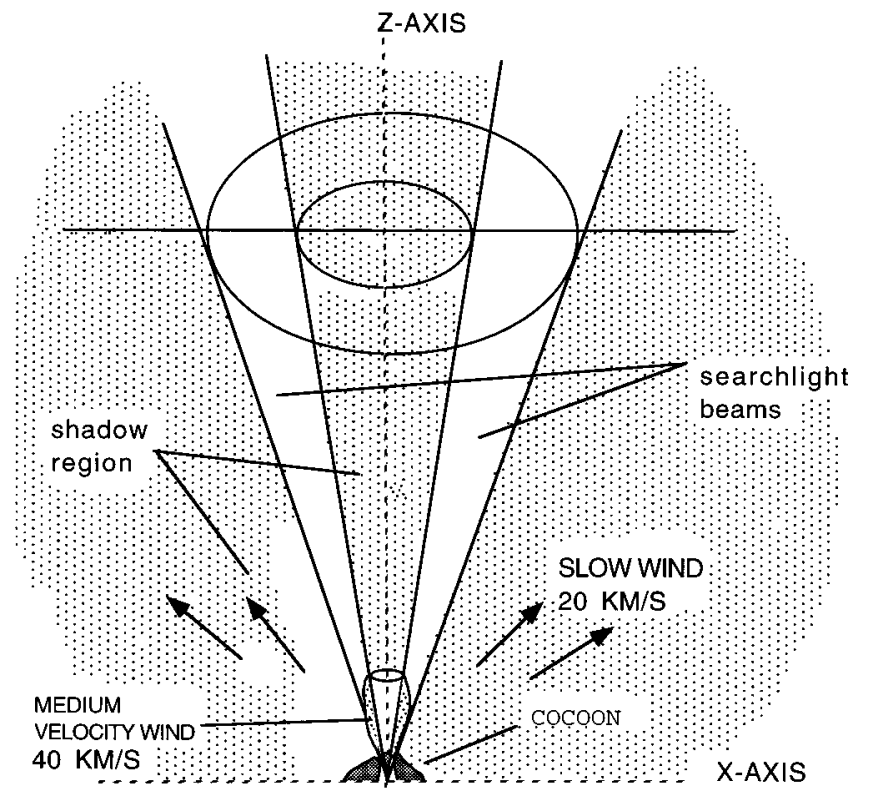
2688. 
The detailed morphology of the interface of the dark lane with the northern and southern lobes seen in Figure $1 c$ is precisely what one would expect in our model. Because the cocoon is tilted toward us in the south, the outer regions of the cocoon obscure an extended part of the bright southern lobe, resulting in the extended edge which bulges slightly away from the center of the nebula. In contrast, in the north, we see the polar region where the starlight as well as the high-velocity outflow emerges from the cocoon, which looks like a $W$-shaped apex. The fact that this apex has a W-shaped structure rather than a V-shaped one supports our idea that the holes through which starlight is streaming out have central plugs, i.e., they are annular in shape.

We suggest that these holes have been carved out by a wobbling, high-speed stream of matter; they will play a crucial role in the shaping of the planetary nebula resulting from the evolution of the CRL 2688 central star. The presence of high-speed material in CRL 2688 is known from observations of millimeter-wave $\mathrm{CO}$ lines (Young et al. 1992; Jaminet et al. 1992). In addition to the main $20 \mathrm{~km}$ $\mathrm{s}^{-1}$ molecular outflow, Young et al. (1992) have shown that CRL 2688 has at least two additional winds expanding at higher velocities, one a medium-velocity wind (MVW) expanding at $\sim 40 \mathrm{~km} \mathrm{~s}^{-1}$, and two a high-velocity wind (HVW) expanding at (a projected velocity of) $\sim 100 \mathrm{~km} \mathrm{~s}^{-1}$ ( $320 \mathrm{~km} \mathrm{~s}^{-1}$ deprojected). Very rough estimates of the radial extent of the MVW and HVW are $\sim 10^{\prime \prime}$ and 0.3 (Young et al. 1992). The momentum in the HVW, which is inferred to have a mass-loss rate of $10^{-3} M_{\odot} \mathrm{yr}^{-1}$, is much higher compared to that in the slow wind.

The presence of the MVW and HVW allows a relatively simple explanation for the spindle-shaped structure of the inner region in the context of our model. The expansion of the HVW is constrained by dense material in the intersearchlight region near and along the polar directions. Thus the inward curving boundary of the spindle delineates the boundary of the shocked layer of gas resulting from the hydrodynamic interaction of the HVW with the slow (20 $\mathrm{km} \mathrm{s}^{-1}$ ) one. The MVW is probably not a real wind ejected from or near the star but simply gas in the slow outflow which has been swept up and accelerated by the HVW. The presence of the shadow region which bifurcates the bright spindle, is precisely what we expect if the high-speed gas is streaming out through the same annular holes in the dust cocoon as the radiation which produces the searchlight beams. We expect the shocked gas to be hot, and our expectation is confirmed by the close match (both in location and size) between the bright spindle-shaped lobes in our WFPC2 image (Fig. 1c) and the polar lobes of $2.1 \mu \mathrm{m}$ emission from shocked $\mathrm{H}_{2}$ (LHK93; and recent high-resolution $2 \mu \mathrm{m}$ imaging using the NICMOS/HST camera in Sahai et al. 1998). The southern spindle is less extended than the northern one (by $\approx 15 \%$ ), showing that the HVW has been decelerated to a greater extent in the south, indicating that the southern half of the dust cocoon is thicker than the northern one.

\subsection{The Radial Extinction of Starlight}

We will now set constraints on the radial extinction of starlight by examining the scattered intensity. We first derive the radial scattering optical depth in the CRL 2688 nebula assuming (1) there is no obscuration of starlight from the central F5 Ia star, (2) all of the observed light is due to single scattering. We will assume an isotropic scattering phase function. For an optically thin scattering nebula (i.e., the line-of-sight scattering optical depth, $\tau_{\text {los,s }} \ll 1$ ), the ratio of the observed scattered light intensity $\left(S_{v}\right)$ from any point, $P$, in the nebula, to the incident stellar radiation at $P$, is directly related to the line-of-sight scattering optical depth, $\tau_{\text {los }, s}$, as

$$
\tau_{\text {los }, s}=8 \pi \varphi^{2} S_{v} /\left[L_{v} \exp \left(-\tau_{\text {ext }}\right) / 4 \pi D^{2}\right],
$$

where $L_{v}$ is the stellar luminosity, $D$ is the distance to the nebula, and $\varphi$ and $\exp \left(-\tau_{\text {ext }}\right)$ are, respectively, the angular distance and extinction from the star to $P$ (this expression accounts for the variation of the stellar flux to different points along the line of sight). Thus, if the extinction is negligible $\left(\tau_{\mathrm{ext}} \ll 1\right), \tau_{\mathrm{los}, s}(\varphi)$ can be estimated easily since from the known spectral type of the central star, we can determine

$$
L_{v} / 4 \pi D^{2}=\left(F_{\mathrm{bol}} / \sigma T_{\text {eff }}^{4}\right)\left(2 \pi h c / \lambda^{3}\right)\left[\exp \left(h c / \lambda k T_{\text {eff }}\right)^{-1}\right]^{-1},
$$

where $F_{\text {bol }}$ is the bolometric flux, estimated to be $6 \times 10^{-7}$ $\mathrm{ergs} \mathrm{s}^{-1} \mathrm{~cm}^{-2}$ (Knapp et al. 1993) from ground-based photometry. Inserting the surface brightnesses taken from our $H S T 0.6 \mu \mathrm{m}$ image at a point $P, 8.5$ from the center, and at a latitudinal angle of $71^{\circ}$ (a region where the density structure of the CRL 2688 nebula is not controversial since it avoids the regions near the searchlight beams and the equatorial plane), we find $\tau_{\text {los }, s}=1.1 \times 10^{-3}$, which is much smaller than the estimate derived earlier from longwavelength observations (see $\S 3$ ). The same analysis applied to a $K$-band image (Fig. 4 in LHK93) gives $\tau_{\text {los }, s}=$ $7.8 \times 10^{-3}$ at $2.1 \mu \mathrm{m}$, which is also clearly absurd since for the submicron dust grains believed to exist in the extended nebular regions in CRL 2688 (Jura, Balm, \& Kahane 1995), the scattering opacity in the $0.6-2 \mu \mathrm{m}$ wavelength range should be a very strongly decreasing function of $\lambda\left(\lambda^{-4}\right.$ Jura et al. 1995; Martin \& Rogers 1987). The above two absurdities result from 2 factors. The first factor is substantial radial wavelength-dependent attenuation of the starlight, which (1) significantly decreases the amount of incident starlight reaching the nebula at $0.6 \mu \mathrm{m}$, and (2) increases the ratio of the incident starlight and thus the scattered intensity, at $2.1 \mu \mathrm{m}$ relative to that at $0.6 \mu \mathrm{m}$. The radial extinction optical depth from points in the main nebular lobes to the central star, $\tau_{\text {ext }}(0.6 \mu \mathrm{m})$, must be about $6-7$, in order to increase the derived $0.6 \mu \mathrm{m}$ scattering optical depth to a value consistent with the estimate based on longwavelength observations. The second factor is an additional source of $2 \mu \mathrm{m}$ flux from hot dust close to the central star. Such a source has been found in the protoplanetary nebula M1-92 (Bujarrabal et al. 1998), where ground-based imaging shows a central pointlike source with a $2 \mu \mathrm{m}$ flux 20 times larger than that expected from the $20,000 \mathrm{~K}$ central star illuminating the nebula.

\subsection{Quantitative Modeling of the Searchlight Beams}

In our proposed model for CRL 2688, the observed latitudinal distribution of nebular light is largely an effect of anisotropic illumination of the nebula due to starlight escaping through an appropriately shaped cavity. Stated differently, the brightness variation across the searchlight beams is simply related to the latitudinal variation of radial extinction optical depth, $\tau_{\text {ext }}(\lambda)$, in the dust cocoon around the central star. In our $0.6 \mu \mathrm{m}$ image, the nebular brightness 
decreases steeply from within the searchlight beams to just beyond their outer edges; e.g., the angular cut in intensity plotted in Figure 4 shows that the intensity decreases to one-third its peak value at an offset of $11^{\circ}$ from the peak position (the polar axis corresponds to an angular offset of $39^{\circ}$ in this plot). This result implies that the corresponding change in extinction optical depth across the proposed annular holes in the cocoon is $>1.2$; this number is a lower limit because the significant contribution to the scattered light from regions in front of and behind the brightly illuminated region reduces the contrast between the searchlight and intersearchlight regions (as we show below using detailed modeling).

We have developed a simple model of starlight escaping through holes in the cocoon surrounding the star, being scattered by nebular dust, and producing the searchlight beams. Only single scattering has been taken into account. The observed intensity, integrated along radius from $8^{\prime \prime}-20^{\prime \prime}$, is shown as a function of angular offset for both the north and south lobes in Figures $6 a$ and $6 b$. The angular offset is measured from the horizontal axis of the F606W image shown in Figure 1. In these plots, the polar axis of the nebula corresponds to the local minimum between the two peaks. Note that the contrast factor in the intensity between the peaks $\left(I_{p}\right)$ and the center $\left(I_{c}\right)$ is quite large, about a factor 2 or more. The presence of the holes in the cocoon will result in an illumination which varies with angular offset from the polar axis, peaking in the center of the holes and decreasing monotonically away from it. We first examined a model in which the illumination function (defined as the stellar intensity emerging from the dust cocoon, with its maximum value normalized to unity) is cylindrically symmetric around the polar axis. The model illumination function and the intensity (model A) is shown overlaid on the data in Figure $6 a$. We find that although our model fits the data fairly well outside the searchlight-beam region, it is not able to produce the observed ratio of intensities between the peaks and the center $\left(I_{p} / I_{c}\right)$; the central region (near offset $=39^{\circ}$ ) is not sufficiently dark in the model. It is not possible to make the central region any darker in the model since the illumination of the central region is already negli-



FIG. $6 a$ gible compared to that in the peak. Thus the contrast in the brightness of the searchlight and intersearchlight-beam region depends only on the difference in the scattering pathlength traversing the searchlight-beam cone along a tangential vector as opposed to a radial vector. We conclude that the hole in the cocoon through which the starlight escapes is not uniform, but has an azimuthal dependence around the polar axis. Defining the azimuthal angle, $\psi$, to be $90^{\circ}$ in the plane of the sky, and zero along the line-of-sight, we find that a model in which the illuminating function decreases by a factor 2 from $\psi=90^{\circ}$ to $\psi=0^{\circ}$ (Model B in Fig. $6 a$ ) is able to fit the data. We have not attempted to fine-tune the illumination function to improve the fits of the models to the data since our goal is only to show that the "annularhole" hypothesis has reasonable quantitative consistency with the data. Although selecting such an azimuthally varying illuminating function requires a preferred orientation, the $K$-band image of LHK93 provides independent support for such an orientation. This image shows that the $\mathrm{H}_{2} S(1)$ emission is concentrated around $\psi= \pm 90^{\circ}$. If the cocoon is relatively thinner around $\psi= \pm 90^{\circ}$, as our modeling indicates, we would indeed expect the preferred directions for the escape of the high-velocity wind (which shocks surrounding dense gas to produce the $2 \mu \mathrm{m} \mathrm{H}_{2}$ emission) to cluster around $\psi= \pm 90^{\circ}$.

\subsection{The Dust Cocoon}

We now investigate the properties of the dust cocoon obscuring the central star. We can use the illumination function to set constraints on the variation of the radial extinction in the dust cocoon, $\tau_{\text {ext,c }}(0.6 \mu \mathrm{m})$, as a function of offset from the symmetry axis. For example, at an offset of $19^{\circ}$, the illumination function has a value $\approx 0.1$, implying an increase in $\tau_{\mathrm{ext}, c}$ of about 2.3 from $\theta=0^{\circ}$, the peak of the searchlight beam. Assuming the absorption opacity $\kappa_{a}(\lambda) \propto$ $\lambda^{-1}$, the scattering opacity $\kappa_{s}(\lambda) \propto \lambda^{-4}$, and the $0.6 \mu \mathrm{m}$ albedo to be 0.57 , as derived by Martin \& Rogers (1987) for small (radius $a=0.1 \mu \mathrm{m}$ ) amorphous carbon grains, we find that the corresponding increase in the total $2 \mu \mathrm{m}$ extinction of the dust cocoon is $0.3(0.4$ if $a=0.2 \mu \mathrm{m})$. This is too small an increase since the similar-looking latitudinal brightness



FIG. $6 b$

FIG. 6.-Observed intensity (solid curve) integrated along radius from $8^{\prime \prime}-20^{\prime \prime}$ shown as a function of angular offset, for the (a) northern lobe and (b) southern lobe. The angular offset is measured from the horizontal axis of the F606W image shown in Figs. 1a. The polar axis of the nebula corresponds to the local minimum between the two peaks. The model intensity (dashed line) assuming an azimuthally symmetric illumination function (dotted line) is overlaid on the data in Fig. $6 a$ (model A). This model is not able to produce the large observed ratio of intensities between the peaks and the center. It is necessary to use an illuminating function which has an azimuthal dependence around the polar axis in order to fit the data. Model B (dash-dotted curve) shows the intensity using an illuminating function which decreases linearly by a factor 2 from the azimuthal direction lying in the plane of the sky to one perpendicular to it. 
distribution at 2 and $0.6 \mu \mathrm{m}$ (LHK93) imply comparable variations in the extinction due to the cocoon at 2 and 0.6 $\mu \mathrm{m}$ (otherwise the morphology of the nebula at $2 \mu \mathrm{m}$ will appear significantly more round than observed, e.g., Fig. 12 of LHK93). We require variations in $\tau_{\text {ext, c }}(2 \mu \mathrm{m})$ of at least $\sim 1$ in order to produce searchlight-beam-like features at 2 $\mu \mathrm{m}$. Since the exponent for the weakest wavelength dependence of $\kappa_{a}$ for small grains is, in general, $\approx 1$ and, in particular for CRL 2688, is measured to be $\approx 0.8$, modification of the grain properties in the nebula does not appear to be a plausible way of increasing $\tau_{\mathrm{ext}, c}(2 \mu \mathrm{m})$.

As a way out of the above predicament, we are naturally led to the conclusion that there is a different grain component in the cocoon around the central star which has a significantly higher $\kappa_{a}(2 \mu \mathrm{m}) / \kappa_{a}(0.6 \mu \mathrm{m})$ ratio. The cocoon grain sizes must therefore be comparable to $0.6 \mu \mathrm{m}$. The cocoon must contribute a significant fraction (greater than $\sim 2$ ) of the total radial extinction optical depth estimated in our models above. Variations in the latitudinal distribution of the thickness of the cocoon then result in similar latitudinal variations in the illumination of the nebula at both at 0.6 and $2 \mu \mathrm{m}$, resulting in the very similar nebular morphologies at both wavelengths. A precise measurement of the brightness contrast of the searchlight beams at $2 \mu \mathrm{m}$ using, e.g., NICMOS instrument aboard HST, will allow us to calculate the variation in the $2 \mu \mathrm{m}$ radial extinction responsible for the beams; comparison of this with the corresponding $0.6 \mu \mathrm{m}$ extinction determined above from our WFPC2 image will allow us to directly set a lower limit on the sizes of the big grains in the dust cocoon.

From the absence of a pointlike stellar source in the dark lane in our image, we can estimate the $0.6 \mu$ m radial extinction optical depth toward the central star $\left(\tau_{0}\right)$ along directions close to the equatorial plane. With no dust attenuation, the F5Ia central star would produce $2.3 \times 10^{8}$ $e^{-} \mathrm{s}^{-1}$ in WFPC2; since the $3 \sigma$ upper limit on a point-like source at the center of the nebula is $0.68 e^{-} \mathrm{s}^{-1}$, our $3 \sigma$ lower limit for $\tau_{0}$ is 19.6. Because the total radial extinction which arises in the largely spherically symmetric AGB wind, is at most 5 (see $\S 5.1$ ), the remaining extinction must arise in the cocoon, suggesting that the cocoon is much thicker in the equatorial region relative to the polar region.

Why is the northern lobe in CRL 2688 a factor 4-5 times brighter than the southern lobe? There are two possibilities. The first, incorporated in the YMW model, is that the equatorial region is optically thick and since the north lobe is tilted toward us, scattered light from the south lobe (but not from the north lobe) passes through this region and is attenuated; however, this requires not only that the dense equatorial region be at least as extended as the nebula, but that it also have the same attenuation factor at all radii since the southern lobe is fainter than the northern one by roughly the same factor at all radii. We consider that it is more likely that the southern half of the dust cocoon surrounding the star is optically thicker than the northern half, consistent with our suggestion in $\S 5$.

\section{DISCUSSION}

CRL 2688 has entered the protoplanetary phase of evolution very recently. The ejection of the high-velocity wind is believed to signal the end of AGB evolution. Using the linear extent of the bright inner nebula $\left(9 \times 10^{16} \mathrm{~cm}\right)$ and assuming that material in HVW starting out with a speed of $300 \mathrm{~km} \mathrm{~s}^{-1}$ is slowed down to $40 \mathrm{~km} \mathrm{~s}^{-1}$, we estimate that the HVW has been operating for slightly less than $200 \mathrm{yr}$. Are there other objects which appear similar to CRL 2688? The young planetary nebula J900 shows radial beamlike structures (Shupe et al. 1995) which look remarkably similar in their appearance to the searchlight beams seen in CRL 2688. These radial structures are seen prominently in a wide $K$-band image, as well as in a continuum-subtracted $\mathrm{H}_{2} S(1)$ $2.1 \mu \mathrm{m}$ emission-line image. It appears plausible that the dust cocoon surrounding the central star of J900 is very similar to that of CRL 2688. The searchlight-beam like features in the $K$-band image then result from anisotropic illumination of the J900 nebula. The concentration of the $\mathrm{H}_{2}$ $S(1)$ emission in the same region as the $K$-band searchlight beams could result from either one or both of the following: (1) fluorescent excitation by ultraviolet radiation preferentially streaming into this region, and/or (2) a hot, fast stellar wind streaming preferentially into this region and producing the $S(1)$ emission through interaction with the surrounding denser nebular gas.

\subsection{The Origin of the Dust Cocoon}

Although much more work needs to be done to fully characterize CRL 2688, we believe that the observational evidence heavily favors the existence of a dust cocoon structure which is physically different from the extended nebula. Our argument that the grains in the cocoon are larger than in the nebula supports this viewpoint. However, at present we do not know what mechanism is responsible for producing the cocoon or ejecting the high-speed jets. Several different hypotheses (Morris 1990; Livio 1993) have been proposed, all of which directly or indirectly require the central star to be a binary. Soker \& Livio (1994) have examined three mechanisms that can produce collimated outflows, each of which requires the central star to be a binary, with the collimated outflow resulting from an accretion disk. The most likely of these three involves a common envelope binary with a main-sequence secondary. At the end of the common envelope phase, the secondary ejects most of the matter it has accreted, which forms a disk around the primary. Livio \& Pringle (1996) find that the accretion disk powering the outflow is unstable to radiation-induced self-warping instability, which produces a wobbling jet. CRL 2688 fits this picture very well: the cocoon which obscures the central star is the ejected common envelope and the annular holes in the cocoon through which the searchlight beams shine out have been carved out by a high-speed, precessing, collimated jet. Direct evidence for such collimated jets and an accretion disk in a proto-planetary nebula has been uncovered through ground-based adaptive optics imaging of the Frosty Leo Nebula (Roddier et al. 1995). The close (but not precise) linear continuity of the 31.4 (46.1) beam with the $212^{\circ} .1$ (230.9) one in CRL 2688 constrains the northern and southern components of the high-speed bipolar outflow to have correspondingly well-matched opening angles and flow axes.

Bipolar reflection nebulae, which have been observed both around post-AGB stars and pre-main-sequence stars, have traditionally been interpreted in terms of the YMW model (Morris 1981). The findings in this paper raise the possibility that this may not be the correct model in general. It would be worth testing if a model of the type we propose in this paper can explain bipolar reflection nebulae in general. 


\subsection{The Origin of the Arcs}

CRL 2688 is not unique in showing arclike structures; arcs have been seen in scattered light in the neutral circumstellar region of the young planetary nebula NGC 7027 (Bond 1996) and the AGB star IRC + 10216 (Crabtree, McLaren, \& Christian 1987), both of which, like CRL 2688, are carbon-rich and have high AGB mass-loss rates. The Etched Hourglass nebula, MyCn18, another young planetary nebula, shows axisymmetric arclike structures in $\mathrm{H} \alpha$ and $[\mathrm{N} \mathrm{II}]$ which may be the intersection of the expanding ionized bubble with spherical discrete shells in a surrounding neutral envelope (Sahai et al. 1995). Note, however, that arcs will dissipate quickly once they are ionized because of the much larger sound speed at the high temperatures $\left(\sim 10^{4} \mathrm{~K}\right)$ of ionized gas. It is too early to assess whether the presence of arcs in AGB envelopes is a common or uncommon occurrence since the high-resolution imaging necessary to reveal such structures has only recently become possible with the HST.

What is the origin of the arcs? There are three distinct classes of conceivable models. In the first class, the arcs result from processes related to instabilities in the properties of the outer stellar layers which share the stellar rotation. In the second class, the arcs result from instabilities in the region where dust condensation and the acceleration of the mass outflow takes place: a region generally believed to extend out to 1-10 stellar radii and which does not share the stellar rotation. The third class of models invoke an external factor, such as the presence of a binary companion. Since most of the arcs do not represent complete shells but typically span a wide range of angular sizes anywhere from about $5^{\circ}$ to $60^{\circ}$, class I models require that the rotation period of the outer stellar layers be larger than the timescales over which the arcs are produced, 75-200 yr, otherwise the resulting shells would describe complete surfaces of revolution around the rotation axis. The morphology of the arcs does not appear to support such a structure. Therefore, the rotation period of the outer stellar layers must be $\geq \sim 500 \mathrm{yr}$, which implies a main-sequence rotation period $\geq \sim 0.5 \mathrm{yr}$, given a typical AGB stellar radius $\approx 1000$ times larger than its main-sequence radius, and assuming no significant loss of angular momentum during main-sequence to AGB evolution.

We now discuss two possible class I models. The first is indicated by a study by Mastrodemos, Morris, \& Castor (1995) showing that the luminosity variations which occur in long-period variables produce periodic density fluctuations in the dust and gas with the same periodicity (typically $\sim 1 \mathrm{yr}$ ). However, since dust streaming and gas-dust viscous heating smooth out these fluctuations at large distances $\left(10^{16} \mathrm{~cm}=0\right.$ ".7 $)$, they speculated that a mechanism which could produce long-period amplitude modulations would give rise to density fluctuations with much longer spatial wavelengths as represented by the arc structure in CRL 2688. Such a mechanism is provided by Icke et al. (1992) who have studied the dynamical response of the outer layers ("mantle") (from where mass loss is presumably generated) of an evolved AGB star to pulsations originating in the stellar interior. The "mantle," driven periodically by pressure waves generated by the motion of the interior regions of the star, represents a "driven oscillator" which exhibits chaotic motions for a wide range of relevant parameters. The most relevant feature of Icke et al.'s theory to our obser- vations of the arcs is that it gives a timescale for variations in the mantle which is in the right ballpark. We use some of the representative solutions for the mantle-radius oscillation amplitude (e.g., Figs. 10 and 11 of Icke et al., bottom four panels) which displays large modulations on timescales corresponding to values of the dimensionless parameter $\tau=t \omega_{m}=50$ to 300 , where $\omega_{m}^{2}=G M_{*} / R_{*}^{3}\left(t=\right.$ time, $M_{*}$ and $R_{*}$ are stellar mass and radius). Substituting appropriate values for $M_{*}\left(1 M_{\odot}\right)$ and $R_{*}\left(8 \times 10^{13} \mathrm{~cm}\right.$, derived from $L_{*}=2 \times 10^{4} L_{\odot}, T_{\text {eff }}=2000 \mathrm{~K}$ ) for CRL 2688 during its AGB mass-loss phase, we find $t \approx 100-600$ yr. Although Icke et al.'s model does not deal with unbound solutions of the equation of motion which lead to mass loss, the expectation is that oscillations with the largest amplitudes will lead to mass loss which will be highest when the amplitude of oscillations is largest. The properties of the expanding AGB wind (mass-loss rate, outflow speed) are thought to result from radiation pressure on dust grains condensing in a very extended atmosphere around the star. Temporal variations in the temperature and density of the mantle gas due to the mechanism described by Icke et al., coupled with nonradial perturbations, could therefore lead to variations in the outflow properties resulting in the shells represented by the arcs.

A second class I mechanism for producing the arcs follows from the suggestion by Schwarzschild (1975) that red giant star atmospheres contain giant convection cells from which mass loss proceeds more efficiently than from other parts of the surface. The gas in the region of the convection cell is cooler than its surroundings, allowing more efficient grain formation, and therefore a higher massloss rate. In this explanation of the arcs, the thickness of the arcs gives us the lifetime for the convection cells (75-200 yr), and their separation provides the timescale over which the cells are regenerated (150-450 yr).

We rule out helium-shell flashes as a possible mechanism for producing the arcs because these are believed to occur on timescales of $\sim 10^{4} \mathrm{yr}$ in AGB stars (e.g., Wood \& Vassiliadis 1993).

For a class II model, we envision that instabilities in the highly nonlinear dust condensation process, occurring in the extended pulsation-driven layers of matter surrounding the stellar surface, result in the presence of regions with enhanced dust grain densities. The mass-loss which ensues from the gas being dragged by grains accelerating outward due to radiation pressure is then higher from these regions. The radial motion of such a locally dense parcel of gas then results in an expanding curved shell with a higher density than its environment. Localized enhancements in the outflow velocity may also lead to the production of such shells, as parcels of gas moving with higher speeds are quickly slowed and compressed into thin sheets by the ram pressure of more slowly moving material in front of them. Quantitative support for this hypothesis would require one to show that the seed instabilities must be produced every 150-450 $\mathrm{yr}$ (the arc separation timescale) and must last about 75-200 yr (the arc thickness timescale).

A possible class III model has been described in a recent paper by Harpaz, Rappaport, \& Soker (1997), in which the periastron passage (occurring every few $100 \mathrm{yr}$ ) of a companion in a highly eccentric orbit around the mass-losing primary diverts mass from the normally isotropic outflow to the equatorial plane, producing the "spacings" between the arcs. The arcs thus represent the isotropic red giant 
wind. This model is attractive because it apparently explains both the appearance of the arcs as well as the dense equatorial cocoon of dust. However, there are several major difficulties with this model. First, it predicts a rather regular arrangement of arcs with their thickness increasing systematically with time, which is not consistent with our image. Their model predicts that over a period of $6,000 \mathrm{yr}$, which corresponds to a radial distance of $24^{\prime \prime}$ in our image, the arcs should systematically increase their thickness by a factor 2, which is not observed. Second, their model does not explain why the arcs show significant departures from circularity, nor why a large fraction of them span small angles.

\section{CONCLUSIONS}

Based on our high-resolution (0"1) wideband $606 \mathrm{~nm}$ image of the proto-planetary nebula, CRL 2688, taken with WFPC2/HST, which shows a pair of radial "searchlight beams" emerging from within a dark lane obscuring the illuminating central star, and a large number of roughly round arcs with their center of curvature in the vicinity of the central star, we conclude the following.

1. The central star in CRL 2688 is surrounded by a thick, equatorially dense cocoon of matter, with starlight escaping through a pair of nonuniform annular holes coaxial with the polar axis. The holes have probably been carved out by the high-velocity outflow detected through millimeter-wave spectroscopy. The polar, conical cavities in the nebular density distribution envisioned in the currently popular model of CRL 2688 do not exist.

2. The cocoon is a compact physical structure distinct from the extended red giant wind which forms the extended nebula. It contains dust grains of size about $0.6 \mu \mathrm{m}$, significantly larger than those found in the extended nebula.

3. The arcs result from incomplete shell-like structures with enhanced densities which correspond to local increases in the mass-loss rate by factors of 2-3. These shells are produced every $150-450 \mathrm{yr}$, over periods of 75-200 yr.

4. The average surface brightness varies as $r^{-3.7}$, implying that the underlying mass-loss rate, or the scattering opacity of the dust grains, varies as $r^{-0.7}$. Independent millimeter-wave ${ }^{13} \mathrm{CO} J=1-0$ observations support the increasing mass-loss rate interpretation.

R. S. thanks Dr. Crabtree for making his ground-based images of CRL 2688 available in digital form. This study was partially funded by the WFPC2 Investigation Definition Team under NASA contract NAS7-1260.
Bieging, J., \& Nguyen-Q-Rieu. 1996, AJ, 112, 706

Biretta, J. A., et al. 1996, WFPC2 Instrument Handbook, version 4.0 (Baltimore: STScI)

Bond, H. 1996, HST press release STScI-PR96-05

Bujarrabal, V., Alcolea, J., Sahai, R., Zamorano, J., \& Zijlstra, A. A. 1998, A\&A, in press

Crabtree, D. R., McLaren, R. A., \& Christian, C. A. 1987, Astrophysics \& Sp. Sci. Lib., 132, 145

Crabtree, D. R., \& Rogers, C. 1991, BAAS, 23, 911

. 1993, in ESO Conf. Proc. 46, Mass Loss on the AGB and Beyond, ed. H. Schwarz (Garching: ESO), 255

Harpaz, A., Rappaport, S., \& Soker, N. 1997, ApJ, 487, 809

Icke, V., Frank, A., \& Heske, A. 1992, A\&A, 258, 341

Jaminet, P. A., Danchi, W. C., Sandell, G., \& Sutton, E. C. 1992, ApJ, 400, 535

Jura, M. 1986, ApJ, 303, 327

Jura, M., Balm, S. P., \& Kahane, C. 1995, ApJ, 453, 721

Jura, M., \& Kroto, H. 1990, ApJ, 351, 222

Kawabe, R., Ishiguro, M., Kasuga, T., Morita, K.-I., Ukita, N., Kobayashi, H., Okumura, S., Fomalont, E., \& Kaifu, N. 1987, ApJ, 314, 322

Knapp, G. R., Bowers, P. F., Young, K., \& Phillips, T. G. 1994, ApJ, 429, L33

Knapp, G. R., Sandell, G., \& Robson, E. I. 1993, ApJS, 88, 173

Latter, W. B., Hora, J. L., Kelly, D. M., Deutsch, L. K., \& Maloney, P. R. 1993, AJ, 106, 260 (LHK93)

Livio, M. 1993, in IAU Symp. 155, Planetary Nebulae, ed. R. Weinberger \& A. Acker (Dordrecht: Kluwer), 279

Livio, M., \& Pringle, J. E. 1996, ApJ, 465, L55

Mastrodemos, N., Morris, M., \& Castor, J. 1996, ApJ, 468, 851

Martin, P. G., \& Rogers, C. 1987, ApJ, 322, 374

Morris, M. 1981, ApJ, 249, 572

\section{REFERENCES}

Morris, M. 1990, in From Miras to Planetary Nebulae: Which Path for Stellar Evolution, ed. M. O. Mennessier \& A. Omont (Gif-sur-Yvette: Editions Frontières), 520

Ney, E. P., Merrill, K. M., Becklin, E. E., Neugebauer, G., \& WynnWilliams, C. G. 1975, ApJ, 198, L129

Roddier, F., Roddier, C., Graves, J. E., \& Northcott, M. J. 1995, ApJ, 443, 249

Sahai, R. 1990, ApJ, 362, 652

Sahai, R., Trauger, J. T., \& Evans, R. W. 1995, BAAS, 27, 1344

Sahai, R., Hines, D. C., Kastner, J. H., Weintraub, D. A., Trauger, J. T., Rieke, M. J., Thompson, R. I., \& Schneider, G. 1998, ApJL, in press

Schwarzschild, M. 1975, ApJ, 195, 137

Shupe, D. L., Armus, L., Matthews, K., \& Soifer, B. T. 1995, AJ, 109, 1173

Soker, N., \& Mario, L. 1994, ApJ, 421, 219

Sopka, R. J., Hildebrand, R., Jaffe, D. T., Gatley, I., Roellig, T., Werner, M., Jura, M., \& Zuckerman, B. 1985, ApJ, 294, 242

Tamura, M., Hasegawa, T., Ukita, N., Gatley, I., McLean, I. S., Burton, M. G., Rayner, J. T., \& McCaughrean, M. J. 1988, ApJ, 326, L17

Trauger, J. T., et al. 1994, ApJ, 435, L3

Truong-Bach, Morris, D., Nguyern-Q-Rieu, \& Deguchi, S. 1990, A\&A, 230, 431

Watson, A. M. 1994, Ph.D. thesis, Univ. Wisconsin, Madison

Wood, P. R., \& Vassiliadis, E. 1993, in IAU Symp. 155, Planetary Nebulae, ed. R. Weinberger \& A. Acker (Dordrecht: Kluwer), 291

Yamamura, I., Onaka, T., Kamijo, F., Deguchi, S., \& Ukita, N. 1995, ApJ, 439, L13

439, 1996, ApJ, 465, 926

Young, K., Serabyn, G., Phillips, T. G., Knapp, G. R., Güsten, R., \& Schulz, A. 1992, ApJ, 385, 265

Yusef-Zadeh, F., Morris, M., \& White, R. L. 1984, ApJ, 278, 186 (YMW) 


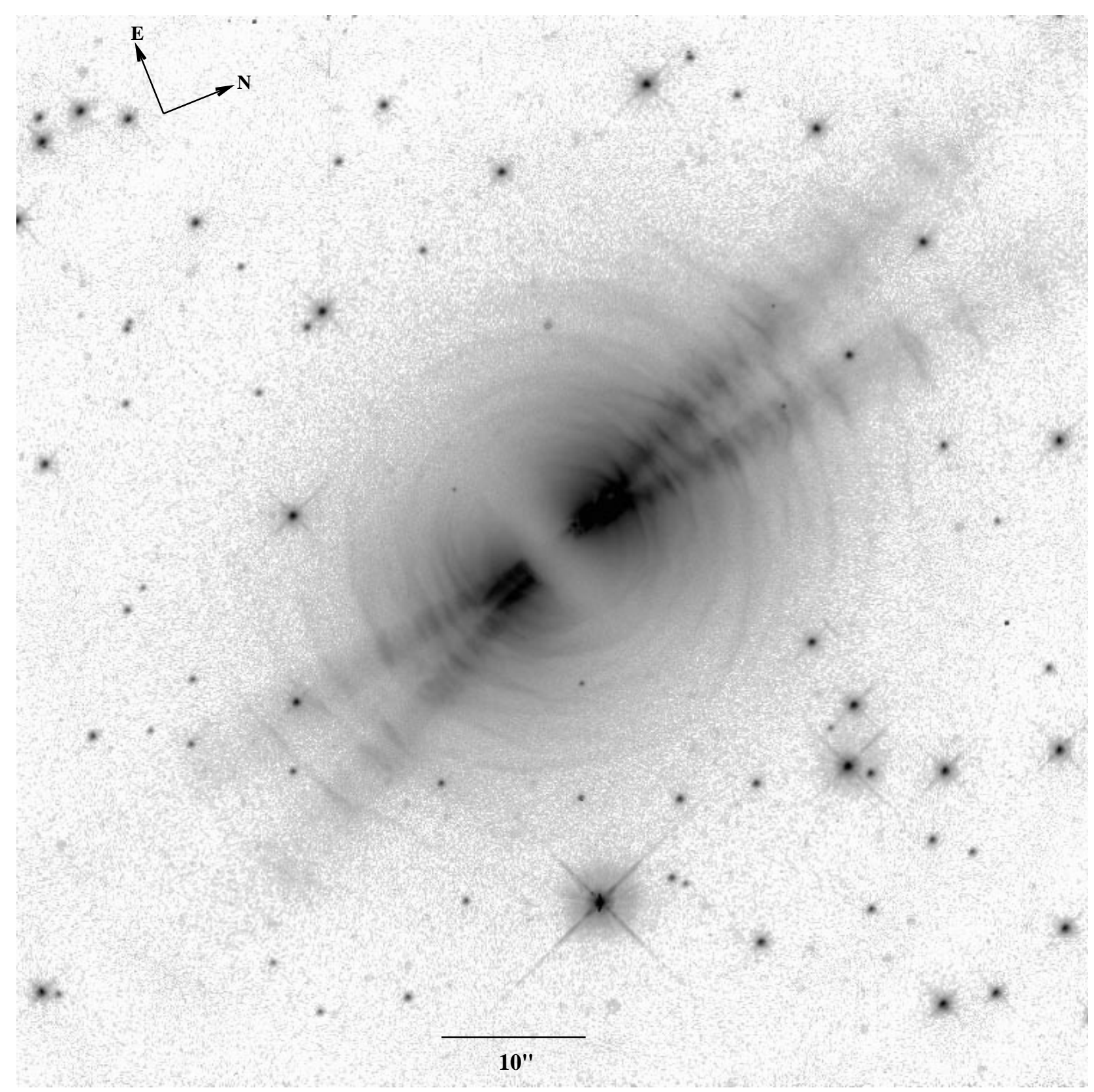

FIG. $1 a$

FIG. 1.- (a) HST Wide Field Camera image (in reverse gray scale) of the proto-planetary nebula CRL 2688 at $606 \mathrm{~nm}$ (taken using the F606W WFPC2 filter). The major axis of the nebula is inclined at $39^{\circ}$ measured counterclockwise from the horizontal axis (which is at a P.A. of $-21: 83$ ). The image size is 74 ".24 $\times 74$ ".24. A logarithmic intensity scale has been used in order to show the structure in both the bright and faint parts of the nebula clearly. The maximum (black) and minimum (white) values of the displayed intensity scale are 15.18 and $22.18 \mathrm{mag} \operatorname{arcsec}^{-2}$, respectively. The location of the obscured central star $\left(\alpha_{\mathrm{J} 2000}=21^{\mathrm{h}} 02^{\mathrm{m}} 18^{\mathrm{s}} .27, \delta_{\mathrm{J} 2000}=36^{\circ} 41^{\prime} 37^{\prime \prime} .0\right)$ is offset $37^{\prime \prime} .05$ along the horizontal axis and $37^{\prime \prime} .05$ along the vertical axis from the bottom left-hand corner of the image. (b) A sharpened version of the image in $(a)$ generated by subtracting an unsharp mask of the image from the original, shown in reverse gray scale. (c) An enlarged view of the inner 14.94 × 14".94 region of CRL 2688 (shown in reverse gray scale), showing that the edges of the brightest emission regions curve inward (seen clearly in the southern lobe but not in the northern lobe because of saturation) and do not follow the straight edges of the extended beams. A logarithmic intensity scale has been used, with the maximum (black) and minimum (white) values of the displayed intensity scale set at 14.93 and $18.68 \mathrm{mag} \operatorname{arcsec}^{-2}$, respectively. The (obscured) central star is offset by 7".57 along the horizontal axis and 7".47 along the vertical axis from the bottom left hand corner of the image.

SAHAI et al. (see 493, 302)

\section{PLATE 8}




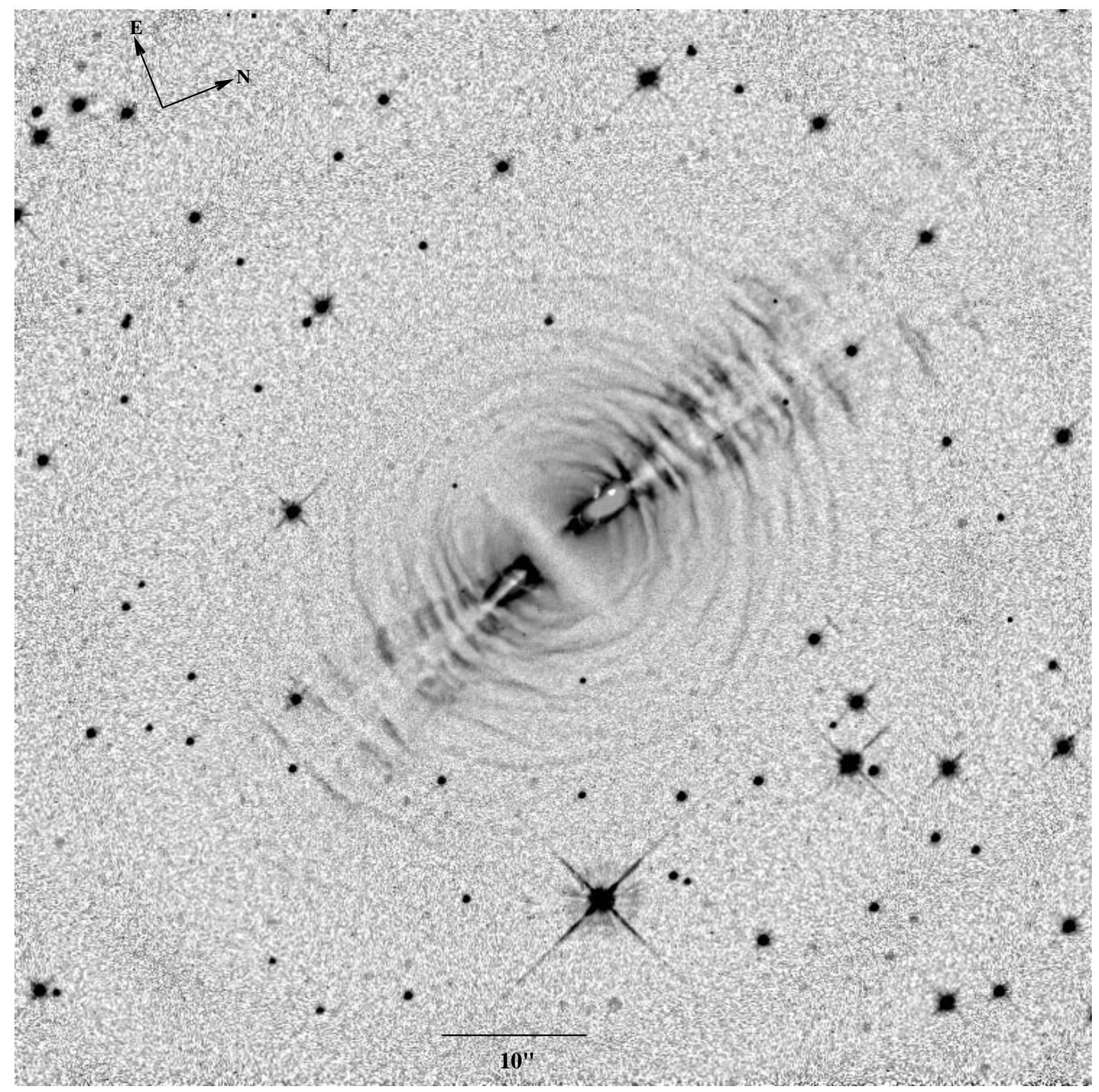

FIG. $1 b$

SAHAI et al. (see 493, 302)

PLATE 9 




FIG. 1c

SAHAI et al. (see 493, 302)

PLATE 10 